\title{
Association of Resilience and Social Networks with Pain Outcomes Among Older Adults
}

\author{
Shirley Musich, PhD, ${ }^{1}$ Shaohung S. Wang, PhD, ${ }^{1}$ Luke Slindee, PharmD, ${ }^{2}$ \\ Sandra Kraemer, MSW, ${ }^{3}$ and Charlotte S. Yeh, MD ${ }^{4}$
}

\begin{abstract}
Depression, stress, and poor sleep have been associated with increased pain among older adults; positive resources, such as resilience and social networks, may help to buffer the impacts of these negative attributes on pain outcomes. The primary objective was to determine the relative effects of positive resources and negative attributes on pain outcomes among older adults with diagnosed back pain, osteoarthritis, and rheumatoid arthritis. The stratified study sample was identified from older adults ages $\geq 65$ years. Members received a survey assessing positive resources (resilience, social networks), negative attributes (depression, stress, poor sleep), and pain outcomes (severity, interference). Opioid and other medication use was determined from pharmaceutical claims. After weighting to representative distributions of pain conditions and adjusting for survey response bias, multinomial logistic regressions were used to determine the relative associations of positive and negative attributes on pain outcomes. Among survey respondents $(\mathrm{N}=4161)$, prevalence of selfreported pain severity and interference for no/mild, moderate, and severe categories was $61 \%, 21 \%$, and $18 \%$, and $67 \%, 16 \%$, and $17 \%$, respectively. In bivariate models, negative attributes of depression, stress, and poor sleep had stronger associations with pain severity and interference than the moderating effects of positive resources of high resilience and diverse social networks. In fully adjusted multivariate models, the strongest associations with moderate and severe pain severity and interference remained depression, stress, and poor sleep. Based on these results, multidimensional pain management strategies should include management of negative attributes along with enhancement of positive resources for effective management of chronic pain.
\end{abstract}

Keywords: older adults, resilience, social networks, pain outcomes

\section{Introduction}

$\mathbf{T}$ He Negative ATTRIBUtes of depression, ${ }^{1-7}$ stress, ${ }^{2,8-10}$ and poor sleep ${ }^{10-17}$ have been associated with increased pain outcomes among older adults. There is general consensus that positive resources, such as resilience and social connectedness, can help to offset some of the impact of these negative attributes on health outcomes. ${ }^{1,2,5,12,18-26}$ Meanwhile, social science research has demonstrated consistent evidence of a negativity bias whereby negative stimuli, (eg, social strain, ambivalent ties, adverse events) influence physiological outcomes more strongly than do positive stimuli or events. ${ }^{12,24,25}$ It was of interest to understand if this phenomenon could be documented within the context of pain outcomes for the protective effects of positive resources relative to the detrimental impacts of negative attributes.

Among negative attributes, depression has been associated most consistently with pain severity and interference. ${ }^{1-8,11,18}$ Most consider the relationship to be bidirectional in that depression is associated with the likelihood of increased pain and pain is associated with the likelihood of increased depression. $^{3,6}$ With the frequent co-occurrence of pain and

\footnotetext{
${ }^{1}$ Research for Aging Populations, Optum, Ann Arbor, Michigan.

${ }^{2}$ Informatics \& Data Science, Optum, Minnetonka, Minnesota.

${ }^{3}$ UnitedHealthcare Medicare \& Retirement, Minneapolis, Minnesota.

${ }^{4}$ AARP Services, Inc., Washington, District of Columbia.
}

(c) Shirley Musich et al. 2019; Published by Mary Ann Liebert, Inc. This Open Access article is distributed under the terms of the Creative Commons License (http://creativecommons.org/licenses/by/4.0), which permits unrestricted use, distribution, and reproduction in any medium, provided the original work is properly cited. 
depression, effective pain management strategies, of necessity, would imply the integration of mental health management. Although stress and pain are less often a focus of older adult research, stress overlaps with depression and shares a similar bidirectional association with pain outcomes. ${ }^{2,8-10}$ Perceived stress can have an independent impact on increasing pain severity and interference, or may augment depression and thus its association with pain. . $^{2,9,9,21,24,26}$

Improvements in sleep have been independently associated with improvements in pain. ${ }^{13}$ Although the relationship is considered bidirectional, positive sleep changes improved pain outcomes more strongly than reduced pain improved sleep outcomes. ${ }^{13}$ Poor sleep, although known to be associated with pain, is most often managed with pharmaceuticals and rarely treated as inherent to pain management or pain intervention strategies. ${ }^{13,15}$ Patients consider sleep improvement to be an important outcome of pain management; however, changes in sleep status, even when present, appear to be predominantly incidental to pain management strategies with little understanding of which factors may be driving the changes. ${ }^{13,15}$

Resilience is defined as a multidimensional construct synonymous with reduced vulnerability and with the ability to adapt to adversity with active coping abilities. ${ }^{19}$ Resilience has been shown to be protective in increasing the capacity to manage pain effectively, especially chronic pain. ${ }^{2,5,19}$ Resilience also appears to buffer depression and stress, which in turn indirectly reduces pain, rather than documented mechanisms for a direct pathway to pain per se. ${ }^{5,18-21}$ Notably, in one population-based study of older adults, widespread pain increased the likelihood of depressive symptoms by a factor of 4; meanwhile, resilience moderated the association by about $12 \% .^{5}$ Thus, although resilience buffered the pain-depression relationship significantly, negativity bias was evident in the stronger detrimental impact of the negative attribute.

Finally, social connectedness is generally measured as perceived social support evaluating the individual's perception of support, whether realized or not. ${ }^{1,2,9,24,25}$ More recently, a more quantitative approach to social networks has been designed as a "count" metric for various types of social experiences (eg, the number of contacts among family, friends, neighbors, religious communities, or clubs and organizations) with more diverse networks across types of social contacts conceived as being more stable and thus more protective. ${ }^{23,26}$ Regardless of the specific measure, however, perceived social support, social participation, or diverse social networks have demonstrated a protective effect on pain severity and subsequent functional abilities. $1,2,7,23-25$ Social support appears to buffer biological reactivity to stressful events and is health promoting either through better adherence to healthier behaviors and compliance with medical regimens or through minimizing psychological processes, such as depression. ${ }^{1,23,24,26}$

No published research studies to date were found that considered the relative impact of negative attributes and subsequent protective impact of positive resources on pain outcomes among older adults with Medicare Supplement plans (ie, Medigap). ${ }^{27}$ In the United States, governmentfunded Medicare covers adults ages 65 and older, as well as those younger than age 65 and disabled. Medicare fee-for- service plans (about $70 \%$ of all Medicare plans) pay about $80 \%$ of medical expenditures for these individuals but offer minimal prescription drug benefits. Those enrolled in these Medicare plans are personally responsible for obtaining additional insurance plans (ie, Medicare Supplement or Medigap plans) to cover the remaining $20 \%$ of medical expenses and more inclusive prescription drug coverage (Medicare Part D plans). Although most (about 90\%) of those with original fee-for-service Medicare coverage have some type of supplemental insurance coverage, about $28 \%$ (currently about 11.8 million adults) have purchased Medigap coverage. ${ }^{27}$ Because this population may differ in demographic, socioeconomic, or health status characteristics from general older adult and/or specifically overall Medicare populations, it was of interest to determine the impact of negative attributes on pain severity and interference and, subsequently, the relative protective effects of positive resources on these same pain outcomes. This study adds to the pain literature by its focus on a relatively large population of older adults and its inclusion of multiple variables for both negative attributes and positive resources and their associations with pain outcomes, controlled for demographic, socioeconomic, health status, and pain-related medications.

Thus, the primary objectives were to (1) estimate the negative associations of depression, perceived stress, and poor sleep on pain severity and interference among older adults with documented pain conditions, and (2) estimate the relative protective effects of high resilience and more diverse social networks on these same pain outcomes. This research was covered under the New England Institutional Review Board \#120160532.

\section{Methods}

\section{Study sample}

In 2016, approximately 5 million Medicare insureds were covered by an AARP ${ }^{\circledR}$ Medicare Supplement plan insured by UnitedHealthcare Insurance Company and UnitedHealthcare Insurance Company of New York for New York certificate holders. These plans are offered in all 50 states, Washington DC, and various US territories. AARP Medicare Supplement insureds with AARP $^{\circledR}$ MedicareRx plans insured through UnitedHealthcare (about $55 \%$ of insureds) at least 65 years of age with a minimum of 12-month continuous medical and drug plan enrollment were used to generate a stratified sample mailing list for a pain-related survey. In addition, insureds must have had at least 1 of the following pain conditions identified from diagnosis codes: back pain (new or continuing), osteoarthritis, or rheumatoid arthritis. Those with cancer, trauma, or drug abuse were excluded. The stratified mailing list of 15,000 was drawn from a possible 327,685 insureds meeting eligibility criteria and included 7500 with back pain, 5000 with osteoarthritis, and 2500 with rheumatoid arthritis. Of survey respondents (4423; 29\% response rate), those younger than age 65 years $(\mathrm{N}=75)$ and those who did not answer the pain severity question $(\mathrm{N}=187)$ were excluded. Thus, the final study population included 4161 survey respondents. Their responses were then weighted to adjust for nonresponse bias and to be representative of those with these pain conditions. This weighted study sample was used for the multivariate regression analyses. 


\section{Pain-related survey}

The mailed survey (54 questions) was developed by UnitedHealthcare to assess various aspects of pain including: negative attributes (depression, perceived stress, sleep quality), positive attributes (resilience, social networks), and pain outcomes of pain severity and pain interference. Other questions included self-reported use of physical therapy as a pain management strategy and body mass index (BMI) as a measure of obesity. The survey was mailed with a 2-month window to the stratified sample in May 2018 with a repeat mailing in June 2018 to those who had not yet responded.

\section{Pain outcomes}

Pain severity and pain interference were assessed using the validated 3-item PEG assessment scale. ${ }^{28}$ The 3 items assessed pain severity $(\mathrm{P})$, interference with enjoyment of life $(\mathrm{E})$, and interference with general activity $(\mathrm{G})$ on $0-10$ scales. The 2 pain interference scales were averaged. Pain severity and pain interference were then categorized as no/mild (0-4), moderate (5-6), and severe (7-10). Because pain severity and pain interference were the intended outcome measures, those survey respondents missing pain severity or pain interference were excluded.

\section{Depression}

Depression was measured using the self-reported Patient Health Quesionnaire-2 (PHQ-2). ${ }^{29}$ This 2-item depression screening tool is well validated and used frequently in clinical settings. The 4-level responses were scored 0 (not at all) to 3 (nearly every day) for a total score range of 0 to 6 . The score was then dichotomized as 0-2 (not depressed) and 3-6 (depressed).

\section{Perceived stress}

Perceived stress was measured using the Cohen perceived stress 4-item scale (PSS-4). ${ }^{30}$ Questions 1 and 4 were scored 0 (never) to 4 (very often) and questions 2 and 3 were reverse scored as 4 (never) to 0 (very often). To calculate a perceived stress score, respondents must have answered 2 of the 4 questions. Responses were averaged across the answered questions for a total score of 0 to 4 . Average responses were then dichotomized as 0-2 (never to sometimes) as low stress and 3-4 (fairly often/very often) as high stress.

\section{Sleep quality: Pittsburgh Sleep Quality Index}

Sleep quality was measured using the Pittsburgh Sleep Quality Index. ${ }^{31}$ For this study, the single-item sleep quality question (component 1) was used, with 4 responses from very good to very bad. The responses were dichotomized as good sleep (very good/fairly good) and poor sleep (fairly bad/very bad).

\section{Resilience}

Resilience was measured using the 6-item Brief Resilience Scale. ${ }^{32}$ Responses, ranging 1 to 5 , were scored if at least 3 of the 6 questions were completed and were averaged across the questions answered to give a range of scores from 1 to 5. Resilience was then dichotomized as follows: low (scores 1-3; responses strongly disagree to neutral); and high (scores 4-5; responses agree and strongly agree).

\section{Social network index}

The Social Network Index counts the number of contacts across 4 different types of social connectedness: talking to friends, family or neighbors on the telephone per week; getting together with friends or relatives per week; attending church or religious services per month; attending meeting of clubs or organizations per month. ${ }^{26}$ Available responses included never, 1-2, 3-4, or 5+ times. Additionally, the index included being married (yes/no) scored 1/0. Respondents must have answered at least 3 of the possible 5 questions. Responses were scored 0 to 3 for the social connectedness items and 0 or 1 for the married question for a total score of 0 to 13 . The Aung et $\mathrm{al}^{23}$ study was used as a guideline to categorize the social network index to 3 levels: limited (0-4), medium (5-7), and diverse (8-13).

\section{Pain-related medications}

Opioids were identified from National Drug Codes (NDCs). Days of supply for the 1 year prior to the survey were calculated from prescriptions recorded in the pharmaceutical drug database. Based on the Healthcare Effectiveness Data and Information Set recommendation for use of opioids no longer than 14 days, opioid use was categorized as 0 to $\leq 14$ days (low risk) and $\geq 15$ days (high risk). ${ }^{33}$

Opioids also were categorized into 6 mutually exclusive categories based on US Drug Enforcement Administration opioid drug schedules for acceptability of medical use and potential for abuse or dependency. Those categories are: (1) long acting; (2) short acting, other Schedule II; (3) short acting, oxycodone; (4) short acting, hydrocodone; (5) short acting, Schedule III-IV and nalbuphine; and (6) tramadol. ${ }^{34}$

Other medications often used concurrently with opioids to manage pain included other pain medications (nonsteroidal anti-inflammatory drugs [NSAIDs] and muscle relaxants) and mental health medications (benzodiazepines). Benzodiazepines, muscle relaxants, and NSAIDs were defined from NDCs included in the drug classes for general use benzodiazepines, muscle relaxants, or NSAIDs.

\section{Covariates}

Covariates were included to characterize categories of pain severity and pain interference and to adjust for other risk factors. These covariates included measures of demographics, socioeconomic factors, health status, and other characteristics taken from health plan eligibility and administrative medical claims.

Demographic questions included age and sex. Age groups were defined as: $65-69 ; 70-74 ; 75-79 ; 80-84$; and $\geq 85$ years. Geographical location (Northeast, South, Midwest or West); metropolitan area (urban or other); and low (less than $15 \%$ nonwhite), medium ( $15 \%$ to $59 \%$ nonwhite), and high ( $\geq 60 \%$ nonwhite) minority areas were geocoded from zip codes as determined by the US Census Bureau. AARP Medicare Supplement plan types were grouped by costsharing levels, including high-level coverage plans with minimal co-payments or deductibles, medium-level coverage plans with relatively more co-payments or deductibles, 
and all other plans. A measure of health services access was calculated as primary care physicians (PCPs) per 100,000 capita. Level of medical services utilization from medical claims was calculated as the Hierarchical Condition Category (HCC) score. ${ }^{35}$ This score is used by the Centers for Medicare \& Medicaid Services to risk adjust medical payments across various medical plans according to the health status of the different insured populations. HCC subgroups were defined as follows and utilized to control for health status: HCC scores <0.5, HCC scores 0.5 to $<1.2$, HCC scores 1.2 to $<2.8$, and HCC scores $\geq 2.8$. Physical therapy sessions were identified from self-reported utilization as a strategy used for pain management. BMI was calculated from self-reported weight and height: weight in kilograms/height in meters squared. Obesity was defined as BMI $\geq 30$.

\section{Statistics}

Weighting to adjust for survey nonresponse bias and stratified sampling. Propensity weighting was used to adjust for potential selection bias often associated with survey response to enhance the generalizability of these findings. The propensity weighting utilized available information about the aforementioned demographic, socioeconomic, and health status variables that could potentially influence survey response. The utility of such propensity weighting models to adjust for external validity threats is described elsewhere. ${ }^{36,37}$ In addition, survey responses were weighted to achieve national representation of the AARP Medicare Supplement population with defined pain conditions of back pain, osteoarthritis, and rheumatoid arthritis and 1 year of medical and drug plan eligibility.

Demographics and logistic regression models. Demographic variables were unilaterally tested across pain severity and pain interference categories using chi-square or $t$ tests for categorical or continuous variables, respectively. Characteristics associated with pain categories comparing moderate or severe pain severity or pain interference to no/mild pain severity or pain interference, respectively, were determined using multinomial logistic regression models. Because depression and high stress were strongly correlated ( $>0.60)$, separate models were developed for depression and high stress. The study team initially established a base model that included demographic (age, sex, minority status, location, region), socioeconomic (plan type), access to care (PCPs per 100,000), and health status (HCC score categories, obesity, pain conditions of back pain, osteoarthritis, and rheumatoid arthritis) variables listed in Tables 1 and 2. The team subsequently added each of the psychosocial, medication, and physical therapy variables listed in Tables 3 and 4 one at a time (adjusted bivariate odds ratios [ORs]) and then all variables in full models for depression and stress separately (full model ORs). All analyses were completed using SAS Enterprise Guide Version 7.1 (SAS Institute Inc., Cary, NC, USA).

\section{Results}

Overall, 4423 AARP Medicare Supplement insureds responded to the survey (29\% response rate). Of these, $94 \%$ $(\mathrm{N}=4161)$ met eligibility criteria and were included in the study. Responses were subsequently weighted to a nationally representative population of 308,443 insureds with pain conditions. The distribution of pain conditions among survey respondents were $56 \%, 56 \%$, and $19 \%$ for back pain, osteoarthritis and rheumatoid arthritis, respectively; the weighted distributions were $29 \%, 77 \%$, and $8 \%$, respectively. Despite the differences in pain condition ratios, the weighting resulted in minimal differences in the prevalence of the pain severity or pain interference categories or of any of the variables listed in Tables 1 and 2; consequently, only the survey responses are shown. Survey respondents for pain severity $(\mathrm{N}=4161)$ and pain interference $(\mathrm{N}=4146)$ were $84 \%$ correlated; hence, demographics for the 2 outcomes were similar. Survey respondents for severity and interference outcomes were mostly female, 70-74 years of age, and white (Tables 1 and 2). The prevalence of HCC health status groups (HCC scores $<0.5$, HCC scores 0.5 to $<1.2$, HCC scores 1.2 to $<2.8$, and HCC scores $\geq 2.8$ ) were as follows: $21 \%, 46 \%, 29 \%$ and $5 \%$ for both pain severity and interference.

Among survey respondents, the prevalence of no/mild, moderate, and severe pain severity levels were $59 \%, 22 \%$ and $19 \%$, respectively; and $64 \%, 17 \%$ and $19 \%$, respectively, for pain interference (Tables 1 and 2). The prevalence of depression, high stress, and poor sleep were $14 \%$, $8 \%$ and $22 \%$, respectively, for both pain severity and interference. The prevalence of high resilience and diverse social networks were likewise similar for both pain severity and interference. About one third used opioids 15 days or longer; the most common opioids were hydrocodone and tramadol.

\section{Characteristics associated with pain severity levels: moderate and severe vs. no/mild}

Although attenuated by $37 \%$ to $50 \%$ with the addition to the models of positive resources and pain-related medications, depression and high stress maintained the strongest associations with both moderate and severe pain severity (ORs 2.6-3.2 moderate; 4.7 severe) (Table 3). Poor sleep, attenuated by $20 \%$ to $35 \%$, had the second highest impact. High resilience attenuated moderate and severe pain severity by $40 \%-60 \%$; diverse social networks by $30 \%-40 \%$; and medium social networks by $10 \%-30 \%$. Opioid use was attenuated by $15 \%$ to $25 \%$; benzodiazepine use by $25 \%-40 \%$. High resilience was relatively more protective for pain severity than social networks, medium or diverse.

\section{Characteristics associated with pain interference levels: moderate and severe vs. no/mild}

As expected, negative attributes and positive resources had similar associations with pain interference as with pain severity. The impact of depression and stress on pain interference was attenuated by $40 \%-50 \%$ with the addition to the models of positive resources and pain-related medications. Nevertheless, depression and high stress maintained the strongest associations with both moderate and severe pain interference (ORs 2.7-3.2 moderate; 6.3-7.0 severe) (Table 4). Poor sleep, attenuated by $20 \%-30 \%$, had the second highest impact. High resilience attenuated moderate and severe pain interference by $50 \%-60 \%$; diverse social networks by $50 \%-60 \%$; and medium social networks by 
Table 1. Unadjusted Demographic Characteristics by Pain Severity Levels

\begin{tabular}{|c|c|c|c|c|c|}
\hline & \multirow[b]{2}{*}{$\begin{array}{l}\text { All mean } \\
\text { or } \%\end{array}$} & \multicolumn{3}{|c|}{ Pain severity } & \multirow[b]{2}{*}{$\mathrm{P}$ value } \\
\hline & & $\begin{array}{l}\text { None/mild }(0-4) \\
\text { mean or } \%\end{array}$ & $\begin{array}{c}\text { Moderate }(5-6) \\
\text { mean or } \%\end{array}$ & $\begin{array}{c}\text { Severe }(7-10) \\
\text { mean or } \%\end{array}$ & \\
\hline Number & 4161 & 2447 & 910 & 804 & \\
\hline \multicolumn{6}{|l|}{ Sex } \\
\hline $\begin{array}{l}\text { Female } \\
\text { Male }\end{array}$ & 67.2 & 63.0 & 73.5 & 72.8 & \multirow[t]{2}{*}{$<0.0001$} \\
\hline Male & 32.8 & 37.0 & 26.5 & 27.2 & \\
\hline Age groups & 75.9 & 75.6 & 76.5 & 75.9 & 0.004 \\
\hline $65-69$ & 21.3 & 22.0 & 19.2 & 21.6 & 0.04 \\
\hline $70-74$ & 25.7 & 26.8 & 23.6 & 25.0 & \\
\hline $75-79$ & 24.7 & 24.9 & 24.1 & 24.8 & \\
\hline $80-84$ & 14.0 & 13.1 & 16.4 & 14.1 & \\
\hline$\geq 85$ & 14.2 & 13.2 & 16.7 & 14.6 & \\
\hline \multicolumn{6}{|l|}{ Minority (from zip codes) } \\
\hline Low & 50.8 & 52.3 & 48.2 & 49.3 & \multirow[t]{3}{*}{0.04} \\
\hline Median & 45.1 & 43.4 & 48.0 & 47.0 & \\
\hline High & 2.9 & 2.9 & 2.5 & 3.4 & \\
\hline \multicolumn{6}{|l|}{ Location } \\
\hline Metro & 83.4 & 83.6 & 85.2 & 80.7 & \multirow{2}{*}{0.04} \\
\hline Other & 16.6 & 16.4 & 14.8 & 19.3 & \\
\hline \multicolumn{6}{|l|}{ Region } \\
\hline Midwest & 24.8 & 25.8 & 23.6 & 22.8 & \multirow{4}{*}{0.17} \\
\hline Northeast & 19.7 & 20.0 & 20.1 & 18.4 & \\
\hline South & 26.7 & 25.2 & 27.5 & 30.5 & \\
\hline \multirow{2}{*}{\multicolumn{6}{|c|}{ Access to health care }} \\
\hline & & & & & \\
\hline \multirow{2}{*}{\multicolumn{6}{|c|}{ Plan type coverage }} \\
\hline & & & & & \\
\hline High & 77.2 & 77.3 & 76.7 & 77.7 & \multirow[t]{3}{*}{0.88} \\
\hline Medium & 2.6 & 2.6 & 2.4 & 3.0 & \\
\hline Other & 20.1 & 20.2 & 20.9 & 19.3 & \\
\hline \multicolumn{6}{|l|}{ HCC Score } \\
\hline$<0.50$ & 20.5 & 25.4 & 15.2 & 11.8 & $<0.0001$ \\
\hline 0.50 to $<1.20$ & 45.7 & 47.6 & 44.2 & 41.8 & \\
\hline 1.20 to $<2.80$ & 28.7 & 23.4 & 35.1 & 37.9 & \\
\hline$\geq 2.8$ & 5.0 & 3.6 & 5.6 & 8.5 & \\
\hline PHQ-2 (Depression) & & & & & \\
\hline $0-2$ & 85.5 & 94.1 & 81.1 & 64.4 & \\
\hline$\geq 3$ & 14.2 & 5.8 & 18.7 & 35.1 & \\
\hline Perceived stress & & & & & \\
\hline Low $(0-1)$ & 57.9 & 69.8 & 46.5 & 34.7 & $<0.0001$ \\
\hline Moderate (2) & 33.4 & 26.6 & 42.0 & 44.3 & \\
\hline High (3-4) & 8.1 & 2.9 & 10.7 & 20.7 & \\
\hline Resilience scale & & & & & \\
\hline Low $(<4)$ & 57.4 & 47.0 & 67.3 & 78.2 & $<0.0001$ \\
\hline $\operatorname{High}(\geq 4)$ & 42.0 & 52.5 & 32.1 & 21.4 & \\
\hline Social Network Index & & & & & \\
\hline Limited $(0-4)$ & 27.1 & 22.2 & 30.6 & 38.1 & $<0.0001$ \\
\hline Medium (5-7) & 42.0 & 42.3 & 41.4 & 41.5 & \\
\hline Diverse $(\geq 8)$ & 26.6 & 31.2 & 22.6 & 16.9 & \\
\hline Pittsburgh Sleep Quality Index & & & & & \\
\hline Good & 77.2 & 86.0 & 70.4 & 58.3 & $<0.0001$ \\
\hline Poor & 21.8 & 13.3 & 28.4 & 40.4 & \\
\hline Opioid days of supply & & & & & \\
\hline None or used $\leq 14$ days & 66.2 & 77.5 & 58.9 & 39.8 & $<0.0001$ \\
\hline$\geq 15$ days & 33.8 & 22.5 & 41.1 & 60.2 & \\
\hline Opioid category (initial) & & & & & \\
\hline 1: Long acting & 3.6 & 1.9 & 3.6 & 8.8 & $<0.0001$ \\
\hline 2: Short acting, other Schedule II & 2.0 & 1.3 & 2.4 & 3.7 & $<0.0001$ \\
\hline 3: Short acting, oxycodone & 14.2 & 11.0 & 14.1 & 24.3 & $<0.0001$ \\
\hline 4: Short acting, hydrocodone & 25.4 & 20.8 & 27.8 & 36.4 & $<0.0001$ \\
\hline 5: Short acting, Schedule III - IV & 5.1 & 4.4 & 5.7 & 6.7 & 0.03 \\
\hline 6: Tramadol & 20.5 & 16.6 & 25.5 & 26.9 & $<0.0001$ \\
\hline
\end{tabular}


TABle 1. (CONTINUED)

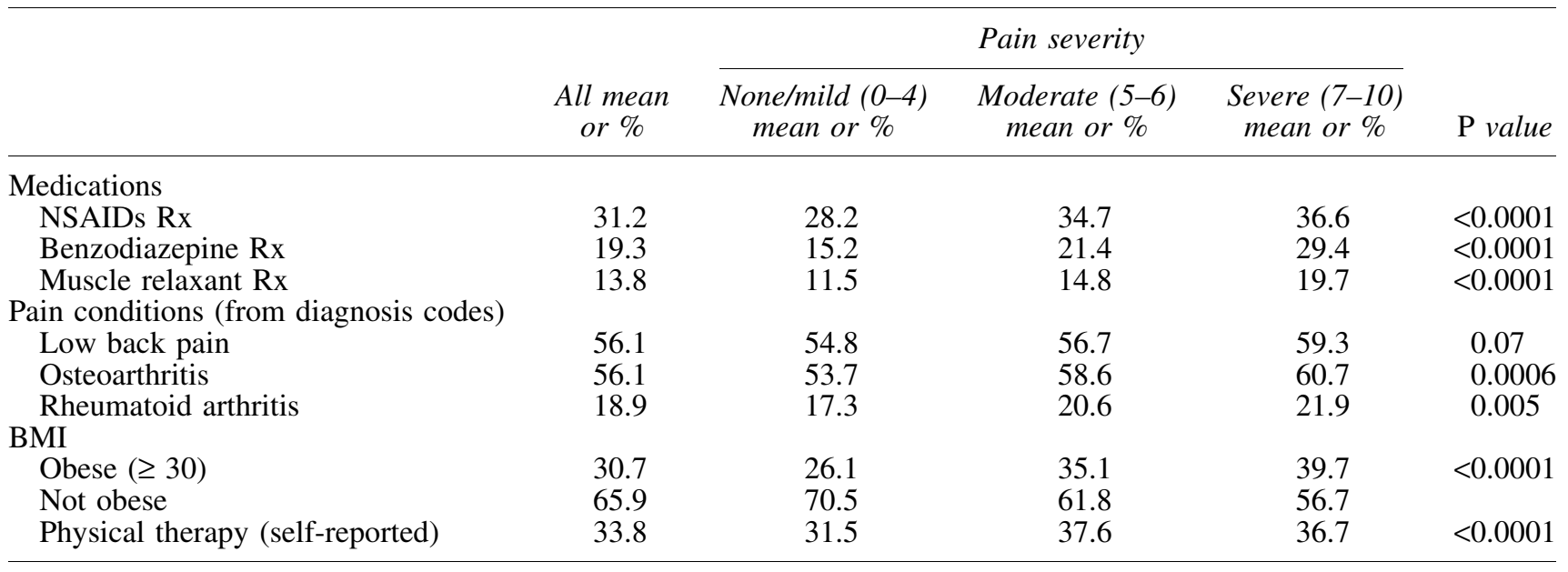

BMI, body mass index; HCC, Hierarchical Condition Category; NSAIDs, nonsteroidal anti-inflammatory drugs; PHQ-2, Patient Health Questionnaire-2; PCP, primary care physician; Rx, prescription.

$30 \%-50 \%$. Opioid use was attenuated by $20 \%-25 \%$; benzodiazepine use by $25 \%-45 \%$. Positive resources, especially medium and diverse social networks, were relatively more protective for pain interference than for pain severity.

\section{Discussion}

In this population of AARP Medicare Supplement insureds, the weighted prevalence of no/mild, moderate, and severe pain severity and pain interference were $61 \%, 21 \%$, and $18 \%$ and $67 \%, 16 \%$, and $17 \%$, respectively. Although measurement scales and definitions of "moderate and severe" pain severity differ somewhat in the literature, the prevalence of pain severity in this population with pain conditions is in general agreement with other studies focused on study populations with chronic pain issues $(62 \%$ $66 \%$ no/mild pain; $34 \%-38 \%$ moderate/severe pain). ${ }^{6,8,22}$

The characteristics most strongly associated with moderate and severe pain severity and interference were depression and stress. ${ }^{2,3,5}$ Although attenuated by positive resources and pain-related medications, ORs associated with moderate and severe pain severity and interference for depression and stress ranged from 3.0-5.0 for pain severity and 3.0-7.0 for pain interference. Of note, stress demonstrated a stronger association with moderate pain than depression. The overall strong associations of depression and stress are consistent with other studies, although the magnitude of the associations were difficult to compare because of differences in study populations, measurement tools for the various scales, and analytic approaches with most studies utilizing total scores as continuous variables in linear regression models. ${ }^{1-7,11}$ The magnitude of the impact for high stress was comparable to that of depression and is especially noteworthy because stress is less often studied in older adult populations.

Sleep was the second strongest characteristic associated with moderate and severe pain severity and interference. ${ }^{10-15,17}$ Despite attenuation by positive resources and pain-related medications, ORs ranged from 2.0-3.0 for moderate and severe pain severity and 3.0-4.0 for moderate and severe pain interference. In longitudinal studies, improvements in sleep have predicted improvements in pain severity. ${ }^{13}$ However, despite an importance attached to good sleep by patients, sleep is generally treated as an incidental symptom associated with pain and managed with pharmaceuticals. ${ }^{13}$ Poor sleep has been associated with depression as well as with pain; however, management of sleep problems has not been extensively integrated into either pain or depression management interventions. ${ }^{13,15}$

High resilience, medium, and diverse social networks reduced the likelihood of moderate and severe pain severity. Additionally, in fully adjusted models controlling for positive resources and pain-related medications, depression and stress were attenuated by about $50 \%$ and poor sleep by about $30 \%$. The study team could find no other studies that compared the magnitude of attenuation for both resilience and/or social networks on pain severity. Resilience had been shown to attenuate the depression and pain relationship by about $12 \%$, which may indicate a stronger buffering impact in this study population. ${ }^{5,18}$ Social support, although often using different metrics, has been shown to demonstrate a protective effect on depression and stress along with pain severity, consistent with these results. ${ }^{1,2,7,22-26}$ Most research studies, however, use continuous variables for relevant scales in linear regression models so direct comparisons of magnitudes of impact are difficult. ${ }^{1-4,6,10-13,19-23}$ Nevertheless, a consistent negativity bias has been demonstrated previously for social support and health outcomes, with social strain being more powerful than supportive social support on cardiovascular risk factors ${ }^{24}$ and ambivalent relationships outweighing supportive relationships for sleep outcomes. ${ }^{25}$ Thus, although present study results cannot be compared to other studies directly, the negativity bias is clearly evident in the data in the magnitude of the impacts for depression, stress, and poor sleep despite positive resources and pain-related medications.

Likewise, the strongest characteristics associated with moderate and severe pain interference, while attenuated, remained depression, stress, and poor sleep. ${ }^{2,4,8}$ High resilience, medium, and diverse social networks significantly 
Table 2. Unadjusted Demographic Characteristics by Pain Interference Levels

\begin{tabular}{|c|c|c|c|c|c|}
\hline & \multirow[b]{2}{*}{ All } & \multicolumn{3}{|c|}{ Pain interference } & \multirow[b]{2}{*}{$\mathrm{P}$ value } \\
\hline & & $\begin{array}{l}\text { None/mild }(0-4) \\
\text { mean or } \%\end{array}$ & $\begin{array}{c}\text { Moderate }(5-6) \\
\text { mean or } \%\end{array}$ & $\begin{array}{c}\text { Severe }(7-10) \\
\text { mean or } \%\end{array}$ & \\
\hline Number & 4146 & 2663 & 700 & 783 & \\
\hline \multicolumn{6}{|l|}{ Sex } \\
\hline Female & 67.1 & 64.7 & 69.3 & 73.6 & \multirow[t]{2}{*}{$<0.0001$} \\
\hline Male & 32.9 & 35.3 & 30.7 & 26.4 & \\
\hline Age groups & 75.9 & 75.8 & 76.3 & 75.8 & 0.24 \\
\hline $65-69$ & 21.3 & 21.9 & 18.1 & 22.4 & 0.54 \\
\hline $70-74$ & 25.8 & 26.0 & 26.0 & 25.0 & \\
\hline $75-79$ & 24.7 & 24.8 & 25.6 & 23.8 & \\
\hline $80-84$ & 14.0 & 13.6 & 14.6 & 14.8 & \\
\hline$\geq 85$ & 14.2 & 13.9 & 15.7 & 14.1 & \\
\hline \multicolumn{6}{|l|}{ Minority (from zip codes) } \\
\hline Low & 50.8 & 52.0 & 46.3 & 51.1 & \multirow{3}{*}{0.04} \\
\hline Median & 45.1 & 43.7 & 49.4 & 45.9 & \\
\hline High & 2.9 & 2.9 & 3.1 & 2.7 & \\
\hline \multicolumn{6}{|l|}{ Location } \\
\hline Metro & 83.4 & 83.7 & 85.1 & 80.6 & \multirow{2}{*}{0.04} \\
\hline Other & 16.6 & 16.3 & 14.9 & 19.4 & \\
\hline \multicolumn{6}{|l|}{ Region } \\
\hline Midwest & 24.8 & 25.9 & 23.1 & 22.4 & \multirow{4}{*}{0.005} \\
\hline Northeast & 19.7 & 20.7 & 19.4 & 16.7 & \\
\hline South & 26.7 & 25.0 & 28.0 & 31.6 & \\
\hline West & 28.7 & 28.2 & 29.4 & 29.4 & \\
\hline \multicolumn{6}{|l|}{ Access to health care } \\
\hline PCPs per 100,000 & 133.7 & 134.2 & 133.1 & 132.3 & 0.53 \\
\hline Plan type coverage & & & & & \multirow{4}{*}{0.12} \\
\hline High & 77.2 & 76.4 & 77.6 & 79.7 & \\
\hline Medium & 2.6 & 2.5 & 3.6 & 2.3 & \\
\hline Other & 20.1 & 21.1 & 18.9 & 18.0 & \\
\hline \multicolumn{6}{|l|}{ HCC Score } \\
\hline$<0.50$ & 20.6 & 25.1 & 13.3 & 11.9 & \multirow[t]{4}{*}{$<0.0001$} \\
\hline 0.50 to $<1.20$ & 45.7 & 47.4 & 43.6 & 41.6 & \\
\hline 1.20 to $<2.80$ & 28.7 & 24.0 & 37.1 & 37.3 & \\
\hline$\geq 2.8$ & 5.0 & 3.5 & 6.0 & 9.2 & \\
\hline PHQ-2 (Depression) & & & & & \\
\hline $0-2$ & 85.5 & 94.9 & 79.4 & 59.4 & $<0.0001$ \\
\hline$\geq 3$ & 14.3 & 5.0 & 20.4 & 40.2 & \\
\hline Perceived stress & & & & & \\
\hline Low $(0-1)$ & 58.0 & 70.2 & 41.9 & 30.8 & $<0.0001$ \\
\hline Moderate (2) & 33.4 & 26.5 & 45.4 & 46.0 & \\
\hline High $(3-4)$ & 8.1 & 2.7 & 12.0 & 22.9 & \\
\hline Resilience scale & & & & & \\
\hline Low $(<4)$ & 57.5 & 47.4 & 72.4 & 78.3 & $<0.0001$ \\
\hline High $(\geq 4)$ & 42.0 & 52.1 & 27.3 & 21.2 & \\
\hline Social Network Index & & & & & \\
\hline Limited $(0-4)$ & 27.0 & 21.2 & 32.4 & 42.0 & $<0.0001$ \\
\hline Medium (5-7) & 42.0 & 43.0 & 41.0 & 39.7 & \\
\hline Diverse $(\geq 8)$ & 26.6 & 31.3 & 22.0 & 14.7 & \\
\hline Pittsburgh Sleep Quality Index & & & & & \\
\hline Good & 77.3 & 85.8 & 69.0 & 56.1 & $<0.0001$ \\
\hline Poor & 21.8 & 13.5 & 30.0 & 42.8 & \\
\hline Opioid days of supply & & & & & \\
\hline None or used $\leq 14$ days & 66.2 & 76.4 & 58.6 & 38.3 & $<0.0001$ \\
\hline$\geq 15$ days & 33.8 & 23.6 & 41.4 & 61.7 & \\
\hline Opioid category (initial) & & & & & \\
\hline 1: Long acting & 3.6 & 1.9 & 3.9 & 9.3 & $<0.0001$ \\
\hline 2: Short acting, other Schedule II & 2.0 & 1.5 & 2.0 & 3.6 & 0.002 \\
\hline 3: Short acting, oxycodone & 14.2 & 10.6 & 16.7 & 24.0 & $<0.0001$ \\
\hline 4: Short acting, hydrocodone & 25.3 & 21.0 & 28.3 & 37.4 & $<0.0001$ \\
\hline 5: Short acting, Schedule III - IV & 5.2 & 4.5 & 5.3 & 7.2 & 0.01 \\
\hline 6: Tramadol & 20.5 & 17.3 & 25.4 & 27.0 & $<0.0001$ \\
\hline
\end{tabular}


TABle 2. (CONTINUED)

\begin{tabular}{|c|c|c|c|c|c|}
\hline & \multirow[b]{2}{*}{ All } & \multicolumn{3}{|c|}{ Pain interference } & \multirow[b]{2}{*}{$\mathrm{P}$ value } \\
\hline & & $\begin{array}{c}\text { None/mild }(0-4) \\
\text { mean or } \%\end{array}$ & $\begin{array}{c}\text { Moderate }(5-6) \\
\text { mean or } \%\end{array}$ & $\begin{array}{c}\text { Severe }(7-10) \\
\text { mean or } \%\end{array}$ & \\
\hline \multicolumn{6}{|l|}{ Medications } \\
\hline NSAID Rx & 31.2 & 29.0 & 35.1 & 35.5 & 0.0001 \\
\hline Benzodiazepine $\mathrm{Rx}$ & 19.3 & 15.9 & 21.7 & 28.7 & $<0.0001$ \\
\hline Muscle relaxant $\mathrm{Rx}$ & 13.8 & 11.3 & 17.0 & 19.3 & $<0.0001$ \\
\hline \multicolumn{6}{|c|}{ Pain conditions (from diagnosis codes) } \\
\hline Low back pain & 56.1 & 54.3 & 57.4 & 60.8 & 0.004 \\
\hline Osteoarthritis & 56.2 & 54.2 & 59.0 & 60.4 & 0.002 \\
\hline Rheumatoid arthritis & 18.8 & 17.6 & 20.9 & 20.8 & 0.04 \\
\hline \multicolumn{6}{|l|}{ BMI } \\
\hline Obese $(\geq 30)$ & 30.7 & 26.1 & 38.3 & 39.5 & $<0.0001$ \\
\hline Not obese & 66.0 & 70.6 & 58.4 & 57.1 & \\
\hline Physical therapy (self-reported) & 33.8 & 30.7 & 40.0 & 39.0 & $<0.0001$ \\
\hline
\end{tabular}

BMI, body mass index; HCC, Hierarchical Condition Category; NSAIDs, nonsteroidal anti-inflammatory drugs; PHQ-2, Patient Health Questionnaire-2; PCP, primary care physician; Rx, prescription.

reduced the likelihood of moderate and severe pain interference. $^{2,19,22}$ In fully adjusted models, depression and stress were attenuated by about $40 \%-50 \%$ and poor sleep by about $20 \%-30 \%$. In other studies, social support has been associated with better physical quality of life scores, and improved functional abilities ${ }^{1,2,16,23}$; meanwhile, resilience has been associated consistently with reduced depression. ${ }^{2-4,6,7,11}$ Nevertheless, the negative attributes remained more strongly associated with pain interference despite positive resources or pain-related medications. $5,12,18,24,25$

Few research studies focused on survey outcomes for positive resources, negative attributes and pain outcomes

Table 3. Regression Adjusted Odds Ratios for Positive Resources and Negative Attributes Associated With Pain Severity Levels

\begin{tabular}{|c|c|c|c|}
\hline & $\begin{array}{c}\text { Adjusted } * \text { bivariate } \\
\text { odds ratios }\end{array}$ & $\begin{array}{c}\text { Full model } * \text { depression } \\
\text { odds ratios }\end{array}$ & $\begin{array}{l}\text { Full model } * \text { stress } \\
\text { odds ratios }\end{array}$ \\
\hline \multicolumn{4}{|c|}{$\begin{array}{l}\text { Pain level: Moderate vs Mild/None }(\mathrm{N}=64,904 \\
\text { weighted moderate pain severity) }\end{array}$} \\
\hline Depression & 4.1 & 2.6 & - \\
\hline Stress-high & 5.0 & - & 3.2 \\
\hline Poor sleep quality & 2.8 & 2.3 & 2.3 \\
\hline High resilience & 0.5 & 0.6 & 0.6 \\
\hline Medium social network index & 0.7 & 0.9 & 0.9 \\
\hline Diverse social network index & 0.5 & 0.7 & 0.7 \\
\hline Physical therapy & 1.1 & 1.1 & 1.1 \\
\hline Opioid use $\geq 15$ days & 2.2 & 1.9 & 1.9 \\
\hline NSAID Rx & 1.5 & 1.4 & 1.4 \\
\hline Benzodiazepine $\mathrm{Rx}$ & 1.5 & 1.1 & 1.1 \\
\hline Muscle relaxant $\mathrm{Rx}$ & 1.4 & $1.0 * *$ & $1.0 * *$ \\
\hline \multicolumn{4}{|c|}{$\begin{array}{l}\text { Pain level: Severe vs Mild/None }(\mathrm{N}=54,723 \\
\text { weighted severe pain severity) }\end{array}$} \\
\hline Depression & 9.4 & 4.7 & - \\
\hline Stress-high & 9.2 & - & 4.7 \\
\hline Poor sleep quality & 4.7 & 3.1 & 3.3 \\
\hline High resilience & 0.3 & 0.5 & 0.4 \\
\hline Medium social network index & 0.5 & 0.8 & 0.7 \\
\hline Diverse social network index & 0.3 & 0.6 & 0.5 \\
\hline Physical therapy & 1.2 & 1.2 & 1.1 \\
\hline Opioid use $\geq 15$ days & 4.6 & 3.5 & 3.6 \\
\hline NSAID Rx & 1.4 & 1.2 & 1.1 \\
\hline Benzodiazepine $\mathrm{Rx}$ & 2.3 & 1.4 & 1.5 \\
\hline Muscle relaxant $\mathrm{Rx}$ & 2.4 & 1.4 & 1.4 \\
\hline
\end{tabular}

*Adjusted for age, sex, minority, region, location, plan type, PCP access, HCC score, obesity, pain conditions (back pain, osteoarthritis, and rheumatoid arthritis).

**Not significant. All other variables significant $P<0.0001$.

HCC, Hierarchical Condition Category; NSAIDs, nonsteroidal anti-inflammatory drugs; PCP, primary care physician; Rx, prescription. 
Table 4. Regression Adjusted Odds Ratios for Positive Resources and Negative Attributes Associated With Pain Interference Levels

\begin{tabular}{|c|c|c|c|}
\hline & $\begin{array}{l}\text { Adjusted } * \text { bivariate } \\
\text { odds ratios }\end{array}$ & $\begin{array}{c}\text { Full model } * \text { depression } \\
\text { odds ratios }\end{array}$ & $\begin{array}{l}\text { Full model }{ }^{*} \text { stress } \\
\text { odds ratios }\end{array}$ \\
\hline \multicolumn{4}{|c|}{$\begin{array}{l}\text { Pain interference: Moderate vs None/Mild ( } \mathrm{N}=49,644 \\
\text { weighted moderate pain interference) }\end{array}$} \\
\hline Depression & 4.5 & 2.7 & - \\
\hline Stress-high & 5.4 & - & 3.2 \\
\hline Bad sleep quality & 3.0 & 2.4 & 2.5 \\
\hline High resilience & 0.4 & 0.5 & 0.5 \\
\hline Medium social network index & 0.6 & 0.7 & 0.7 \\
\hline Diverse social network index & 0.4 & 0.5 & 0.5 \\
\hline Physical therapy & 1.4 & 1.4 & 1.4 \\
\hline Opioid use $\geq 15$ days & 2.1 & 1.7 & 1.7 \\
\hline NSAID Rx & 1.5 & 1.4 & 1.4 \\
\hline Benzodiazepine $\mathrm{Rx}$ & 1.5 & 1.1 & 1.2 \\
\hline Muscle relaxant $\mathrm{Rx}$ & 1.8 & 1.3 & 1.3 \\
\hline \multicolumn{4}{|c|}{$\begin{array}{l}\text { Pain interference: High vs None/Mild }(\mathrm{N}=52,935 \\
\text { weighted severe pain interference) }\end{array}$} \\
\hline Depression & 13.3 & 7.0 & - \\
\hline Stress-high & 12.2 & - & 6.3 \\
\hline Bad sleep quality & 5.3 & 3.5 & 3.7 \\
\hline High resilience & 0.3 & 0.5 & 0.4 \\
\hline Medium social network index & 0.4 & 0.6 & 0.5 \\
\hline Diverse social network index & 0.2 & 0.4 & 0.4 \\
\hline Physical therapy & 1.4 & 1.4 & 1.3 \\
\hline Opioid use $\geq 15$ days & 4.6 & 3.4 & 3.6 \\
\hline NSAID Rx & 1.4 & 1.2 & 1.2 \\
\hline Benzodiazepine $\mathrm{Rx}$ & 2.2 & 1.2 & 1.3 \\
\hline Muscle relaxant Rx & 2.5 & 1.5 & 1.5 \\
\hline
\end{tabular}

*Adjusted for age, sex, minority, region, location, plan type, PCP access, HCC score, obesity, pain conditions (back pain, osteoarthritis, and rheumatoid arthritis). All variables significant $P<0.0001$.

HCC, Hierarchical Condition Category; NSAIDs, nonsteroidal anti-inflammatory drugs; PCP, primary care physician; Rx, prescription.

have had access to pain-related medication data, either selfreported or from database records. ${ }^{4,5,19,22}$ Thus, the present study is relatively unique in that the study team was able to incorporate the documented use of opioids, benzodiazepines, NSAIDs, and muscle relaxants. Although about 34\% used opioids 15 days or longer, the most common use of hydrocodone and tramadol would indicate the management of low-level chronic pain. As expected, use of pain-related medications was most highly associated with severe pain. Nevertheless, the results would indicate that, despite controlling for medication use and positive resources, these patients continued to self-report both moderate and severe levels of pain severity and interference with associated mental health issues.

First-line treatment protocols for pain management have relied largely on opioids and other pharmaceutical analgesics. ${ }^{33}$ These results would indicate that multidimensional interventions concurrently addressing negative attributesespecially depression, stress, and sleep issues-as well as promoting positive resources also may be required for effective pain management. Non-pharmacological approaches targeting mental health and sleep issues that have demonstrated success among older adults might include cognitive behavioral therapy for pain and/or insomnia, mindfulness for stress and pain, or optimized antidepressant therapy with pain management. ${ }^{13,15,38,39}$ Unfortunately, these programs require additional time and resources not generally available to many physicians. Furthermore, published pain interventions often are based on research studies that involve small study populations and lack generalizability to older adults. Physical therapy, exercise therapy, yoga, and progressive muscle relaxation along with psychological interventions have been recommended, especially for back pain issues, but have not been utilized consistently. ${ }^{7,40}$ In addition, research is needed that considers pain management within a holistic context including not only mental and physical health but also the broader social determinants of health (eg, transportation, access to healthy food, living arrangements). Thus, although non-pharmacological approaches to pain management do exist, limitations of physician time, available program resources, and recommended interventions with documented results have made practical applications difficult. Future research might include alternative models of health care delivery with more inclusive services, such as Medicare Advantage or other integrated care delivery models.

This study has some limitations. The study population of AARP Medicare Supplement insureds may not generalize to all older adults or other Medicare or Medicare Supplement insureds. Pharmacy databases confirmed prescription purchases but the study team had no indication of whether patients actually consumed the drugs as directed. Depression, stress, sleep, resilience, social networks, and pain outcomes were self-reported and may be subject to bias. Strengths of 
the study include a relatively large survey population with information on positive resources, negative attributes and pain-related medications that could be directly compared in multivariate regression models.

\section{Conclusions}

Overall, in this population of Medicare Supplement insureds, about $40 \%$ reported either moderate or severe pain severity or interference. Although resilience and social networks attenuated the negative impacts of depression, stress, and poor sleep, the strongest characteristics associated with moderate and severe pain severity and interference remained the negative attributes. Despite positive resources and controlling for pain-related medications, mental health issues were most strongly associated with increased pain outcomes. Thus, based on these results, multidimensional pain management strategies should include management of depression, stress, and poor sleep along with promotion of positive resources, such as resilience or social connectedness, for effective management of chronic pain.

\section{Author Disclosure Statement}

This work was funded by the AARP-Medicare Supplement Insurance. Drs. Musich, Wang, and Slindee, and Ms. Kraemer are all employed by UnitedHealth Group and have stock with UnitedHealth Group. Dr. Yeh is employed by AARP Services, Inc. However, the authors' compensation was not dependent upon the results obtained in this research, and the investigators retained full independence in the conduct of this research.

\section{References}

1. Tripp DA, Abraham E, Lambert M, et al. Biopsychosocial factors predict quality of life in thoracolumbar spine surgery. Qual Life Res 2017;26:3099-3110.

2. Lopez-Olivo MA, Landon GC, Siff SJ, et al. Psychosocial determinants of outcomes in knee replacement. Ann Rheum Dis 2011;70:1775-1781.

3. Kroenke K, Wu J, Bair MJ, Krebs EE, Damush TM, Tu W. Reciprocal relationship between pain and depression: a 12month longitudinal analysis in primary care. J Pain 2011; 12:964-973.

4. Wolfe F, Michaud K. Predicting depression in rheumatoid arthritis: the signal importance of pain extent and fatigue, and comorbidity. Arthritis Rheum 2009;61:667-673.

5. Bauer H, Emeny RT, Baumert J, Ladwig KH. Resilience moderates the association between chronic pain and depressive symptoms in the elderly. Eur J Pain 2016;20: 1253-1265.

6. Sharpe L, McDonald S, Correia H, et al. Pain severity predicts depressive symptoms over and above individual illnesses and multimorbidity in older adults. BMC Psychiatry $2017 ; 17: 166$.

7. Wilkie R, Blagojevic-Bucknall M, Belcher J, ChewGraham C, Lacey RJ, McBeth J. Widespread pain and depression are key modifiable risk factors associated with reduced social participation in older adults: a prospective cohort study in primary care. Medicine (Baltimore) 2016; 95:e4111.

8. White RS, Jiang J, Hall CB, et al. Higher perceived stress scale scores are associated with higher pain intensity and pain interference levels in older adults. J Am Geriatr Soc 2014;62:2350-2356.

9. Harris ML, Loxton D, Sibbritt DW, Byles JE. The influence of perceived stress on the onset of arthritis in women: findings from the Australian longitudinal study on women's health. Ann Behav Med 2013;46:9-18.

10. Eslami V, Zimmerman ME, Grewal T, Katz M, Lipton RB. Pain grade and sleep disturbance in older adults: evaluation the role of pain, and stress for depressed and non-depressed individuals. Int J Geriatr Psychiatry 2016;31:450-457.

11. Lacey RJ, Strauss VY, Rathod T, et al. Clustering of pain and its associations with health in people aged 50 years and older: cross-sectional results from the North Staffordshire Osteoarthritis Project. BMJ Open 2015;5:e008389.

12. Kent RG, Uchino BN, Cribbet MR, Bowen K, Smith TW. Social relationships and sleep quality. Ann Behav Med 2015;49:912-917.

13. Koffel E, Kroenke K, Bair MJ, Leverty D, Polusny MA, Krebs EE. The bidirectional relationship between sleep complaints and pain: analysis of data from a randomized trial. Health Psychol 2016;35:41-49.

14. Guo J, Liu C, Wang X, Qu Z, Zhang W, Zhang X. Relationships between depression, pain and sleep quality with doctor visits among community-based adults in north-west China. Public Health 2017;147:30-38.

15. Vitiello MV, McCurry SM, Shortreed SM, et al. Short-term improvement in insomnia symptoms predicts long-term improvements in sleep, pain, and fatigue in older adults with comorbid osteoarthritis and insomnia. Pain 2014;155:1547-1554.

16. Lallukka T, Sivertsen B, Kronholm E, Bin YS, Øverland S, Glozier N. Association of sleep duration and sleep quality with the physical, social, and emotional functioning among Australian adults. Sleep Health 2018;4:194-200.

17. Sampaio RA, Sewo Sampaio PY, Yamada M, Tsuboyama $\mathrm{T}$, Arai H. Self-reported quality of sleep is associated with bodily pain, vitality and cognitive impairment in Japanese older adults. Geriatr Gerontol Int 2014;14:628-635.

18. Schure MB, Odden M, Goins RT. The association of resilience with mental and physical health among older American Indians: the Native Elder Care Study. Am Indian Alsk Native Ment Health Res 2013;20:27-41.

19. Ramírez-Maestre C, Esteve R. The role of sex/gender in the experience of pain: resilience, fear, and acceptance as central variables in the adjustment of men and women with chronic pain. J Pain 2014;15:608-618.

20. Ong AD, Zautra AJ, Reid MC. Psychological resilience predicts decreases in pain catastrophizing through positive emotions. Psychol Aging 2010;25:516-523.

21. Ong AD, Bergeman CS, Bisconti TL, Wallace KA. Psychological resilience, positive emotions, and successful adaptation to stress in later life. J Pers Soc Psychol 2006; 91:730-749.

22. Richmond NL, Meyer ML, Hollowell AG, et al. Social support and pain outcomes after trauma exposure among older adults: a multicenter longitudinal study. Clin J Pain 2018;34:366-374.

23. Aung MN, Moolphate S, Aung TN, Katonyoo C, Khamchai $\mathrm{S}$, Wannakrairot $\mathrm{P}$. The social network index and its relation to later-life depression among the elderly aged $\geq 80$ years in Northern Thailand. Clin Interv Aging 2016;11: 1067-1074.

24. Uchino BN. Social support and health: a review of physiological processes potentially underlying links to disease outcomes. J Behav Med 2006;29:377-387. 
25. Uchino BN, Kent de Grey RG, Cronan S. The quality of social networks predicts age-related changes in cardiovascular reactivity to stress. Psychol Aging 2016;31:321-326.

26. Pantell M, Rehkopf D, Jutte D, Syme SL, Balmes J, Adler N. Social isolation: a predictor of mortality comparable to traditional clinical risk factors. Am J Public Health 2013; 103:2056-2062.

27. AHIP Center for Policy and Research. Trends in Medigap Enrollment and Coverage Options, 2015. https://www.ahip .org/wp-content/uploads/2017/05/Medigap_Report_5.1.17 .pdf Accessed October 8, 2018.

28. Krebs EE, Lorenz KA, Bair MJ, et al. Development and initial validation of the PEG, a three-item scale assessing pain intensity and interference. J Gen Intern Med 2009;24: 733-738.

29. Kroenke K, Spitzer RL, Williams JB. The Patient Health Questionnaire-2: validity of a two-item depression screener. Med Care 2003;41:1284-1292.

30. Cohen S, Kamarck T, Mermelstein R. A global measure of perceived stress. J Health Soc Behav 1983;24:385-396.

31. Buysse DJ, Reynolds CF 3rd, Monk TH, Berman SR, Kupfer DJ. The Pittsburgh Sleep Quality Index: a new instrument for psychiatric practice and research. Psychiatry Res 1989;28:193-213.

32. Smith BW, Dalen J, Wiggins K, Tooley E, Christopher P, Bernard J. The brief resilience scale: assessing the ability to bounce back. Int J Behav Med 2008;15:194-200.

33. National Committee for Quality Assurance. HEDIS ${ }^{\circledR}$ Measures Included in the 2018 Quality Rating System (QRS). http://www.ncqa.org/hedis-quality-measurement/ hedis-measures/hedis-2018 Accessed March 16, 2018.

34. United States Drug Enforcement Administration. Drug Schedules. https://www.dea.gov/druginfo/ds.shtml Accessed July 5, 2018.
35. Pope GC, Kautter J, Ingber MJ, Freeman S, Sekar R, Newhart C. Evaluation of the CMS-HCC Risk Adjustment Model. 2011. https://www.cms.gov/Medicare/Health-Plans/ MedicareAdvtgSpecRateStats/downloads/evaluation_risk_ adj_model_2011.pdf Accessed March 3, 2017.

36. Fairies DEL, Haro JM, Obenchain RL, Leon AC. Analysis of observational healthcare data using SAS. Cary, NC: SAS Institute, Inc., 2010.

37. Seeger JD, Williams PL, Walker AM. An application of propensity score matching using claims data. Pharmacoepidemiol Drug Saf 2005;14:465-476.

38. Prins B, Decuypere A, Van Damme S. Effects of mindfulness and distraction on pain depend upon individual differences in pain catastrophizing: an experimental study. Eur J Pain 2014;18:1307-1315.

39. Kroenke K, Bair MJ, Damush TM, et al. Optimized antidepressant therapy and pain self-management in primary care patients with depression and musculoskeletal pain: a randomized controlled trial. JAMA 2009;301:2099-2110.

40. Chou R, Qaseem A, Snow V, et al. Diagnosis and treatment of low back pain: a joint clinical practice guideline from the American College of Physicians and the American Pain Society. Ann Intern Med 2007;147:478-491.

Address correspondence to: Shirley Musich, PhD Research for Aging Populations Optum

315 East Eisenhower Parkway, Suite 305 Ann Arbor, MI 48108

E-mail: shirley.musich@optum.com 